\title{
Journal status and new developments
}

\author{
Michael J. Parnham · John A. Di Battista
}

Published online: 16 December 2012

(C) Springer Basel 2012

As the year 2012 comes to an end, it is worth taking stock of the status of the journal. We have seen a considerable increase in manuscript submissions during the year, particularly from Asia, but with a rejection rate of nearly $70 \%$, the quality of published articles has been sustained, resulting in a further increase in the Impact Factor to 2.11. Readers are encouraged to view the Inflammation Research website on the newly reorganised Springer portal, where downloads are visible and access to individual articles is now easier (http://www.springer.com/11). In keeping with the emphasis of the scope of the journal on mechanisms of inflammation and therapy, published articles continue to cover molecular, cellular, experimental and clinical studies. As with many other journals in the field, we have received an increasing number of submissions on gene polymorphisms relevant to inflammation. To ensure the quality of such articles, we have recently updated our Instructions to Authors to provide guidance.
A major change taking place at the end of this year is that the manuscript submission procedure will be migrating from Manuscript Central to Editorial Manager. This brings the journal in line with most other Springer journals. Editorial Manager is quite comparable to Manuscript Central in terms of basic functionality. It is considered to be more straightforward, intuitive, stable and easier to use. Therefore, effective as of 3 December 2012, the Manuscript Central site of Inflammation Research will no longer accept new submissions, and all authors will be re-directed to the Editorial Manager site. Processing of previously submitted manuscripts, including possible revisions, will continue via the Manuscript Central site until a final decision has been reached. We hope all prospective authors will find the new system an improvement, and we look forward to receiving your submissions in the new year.

M. J. Parnham ( $₫)$

Fraunhofer IME-Projektgruppe TMP,

Präklinische Forschung,

c/o Institut für Klinische Pharmakologie,

(Haus 74/75), Klinikum der Johann,

Wolfgang Goethe-Universität,

Theodor-Stern-Kai 7,

60596 Frankfurt am Main, Germany

e-mail: mjparnham@yahoo.co.uk

\section{J. A. Di Battista}

Division of Rheumatology,

Royal Victoria Hospital,

McGill University Health Center,

687 Pine Avenue W., Suite M11.22,

Montreal, QC H3A 1A1, Canada

e-mail: john.dibattista@mcgill.ca 